\title{
Maximum Utility Function Approach for Detection of Primary User Emulation Attack in Cognitive Radio Networks
}

\author{
Abbas Ali Sharifi \\ Department of Electrical Engineering, University of \\ Bonab, Bonab, Iran
}

\author{
Mohammad Mofarreh-Bonab \\ Department of Electrical Engineering, University of \\ Bonab, Bonab, Iran
}

\begin{abstract}
Inherent nature of Cognitive Radio (CR) networks has imposed some serious threats to wireless communications. One of the common threats is Primary User Emulation Attack (PUEA). In PUEA, some malicious users try to imitate primary signal characteristics and defraud CR users to prevent them from accessing the spectrum holes. As a countermeasure against PUEA, we propose Maximum Utility Detection (MUD) approach. A 3-level hypotheses test is considered and maximum utility criterion is applied to choose the hold hypothesis. Simulation results are provided to indicate the superiority of the proposed MUD method against PUEA compared with conventional method.
\end{abstract}

\section{General Terms}

Security, Spectrum Sensing, Cognitive Radio

\section{Keywords}

Cognitive Radio, Spectrum Sensing, Primary User Emulation Attack, Maximum Utility Detection.

\section{INTRODUCTION}

Cognitive Radio (CR) has been widely adopted as a promising technology to overcome the spectrum scarcity by authorizing CR users to operate opportunistically in the free space of the licensed frequency bands in co-existence of the Primary Users (PUs) [1]. Spectrum sensing, with the aim of finding the idle frequency bands (spectrum holes), is the main function of $\mathrm{CR}$ networks [2,3]. Collaborative Spectrum Sensing (CSS) is known as an effective approach to improve the detection performance [3]. Unfortunately, spectrum sensing process is vulnerable to Primary User Emulation Attack (PUEA) [4]. In PUEA, some malicious users send signal similar to that of PU transmitter and causes the CR users to immediately relinquish the desired frequency band [4]. To mitigate the problem of PUEA, many approaches have been proposed.

In [5], an analytical model of the PUEA is proposed and a lower bound on the probability of a successful attack is achieved. In [6], a Received Signal Strength (RSS)-based localization defense strategy under the PUEA is proposed to determine the location of PUEA by deploying sensor network. Collaborative sensing in the presence of PUEA is investigated in [7], where the Fusion Center (FC) assigns an appropriate weight to each $\mathrm{CR}$ user's sensing measurement and then combines them to maximize detection probability in NeymanPearson (N-P) test. In [8], the authors introduce a smart PUEA which is aware of the PU activity and performs spectrum sensing and sends the fake signals with the desired signal occurrence over special frequency band. The authors also investigated the smart PUEA in [9] which applies the target destructive strategy according to its obtained analysis of the radio environment. In [10], the authors present a comprehensive introduction to PUEA, from the attack motivation and its impact on CR networks, to detection and defense approaches. They propose a two-level databaseassisted detection approach to detect of PUEA. Energy detection and location verification are combined for fast and reliable detection. In [11], we introduce an intelligent PUEA with full knowledge of radio environment and exactly colocated with the PU transmitter and transmit with the same power level. The channel occupancy rate of the PUEA is estimated as attack parameter and then the modified N-P criterion is exploited to improve the CSS performance. We also explore an attack-aware threshold selection approach to combat with the PUEA in [12].

In the current study, without deploying additional sensor network and requiring any prior information about location of the PU transmitter, we propose Maximum Utility Detection (MUD) approach. We consider a 3-level hypotheses test based on channel status and utility function is applied to the MUD scheme. Finally, the hold hypothesis is chosen by comparing utility values in each sensing interval.

\section{SYSTEM MODEL}

The considered system model is a centralized CR network including a PU transmitter, $N$ collaborative CR users, an FC and a PUEA. Each CR user independently conducts its spectrum sensing and then local measurements are sent to the FC to take the global decision about the presence or absence of the licensed PU signal. The network model is shown in Fig. 1.

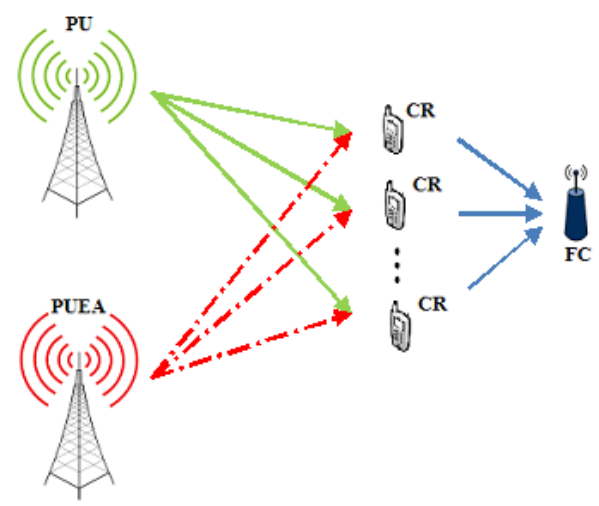

Fig 1. Network Layout

We assume that the energy detection scheme is used for local spectrum sensing. The PUEA is able to perform spectrum sensing to identify the spectrum holes and transmit the fake 
signal to disrupt the $\mathrm{CR}$ network operation. We further assume that the attacker is able to distinguish exactly between occupied and unoccupied frequency bands allocated to the PU. Based on the presence or absence of the PU and PUEA, there are three possibilities which can be expressed as:

$\left\{\begin{array}{lrl}\text { only Noise } & \text { under } & H_{0} \\ \text { PU + Noise } & \text { under } & H_{1} \\ \text { PUEA+ Noise } & \text { under } & H_{2}\end{array}\right.$

The first state $H_{0}$ occurs when the $\mathrm{CR}$ users receive only noise. Moreover, the channel is neither occupied by the PU nor by PUEA. The second state $H_{1}$ happens when the PU transmits over the channel while the PUEA is absent. If the PU is absent and PUEA transmits the fake signal, the CR users receive only the PUEA signal plus noise, as stated by the third hypothesis $H_{2}$. We assume that two hypotheses $H_{1}$ and $H_{0}$ indicate the presence and absence of PU signal, respectively. Similarly, the presence and absence of the PUEA signal are denoted by $E^{o n}$ and $E^{o f f}$, respectively. Based on the above mentioned assumptions, the probability of each hypothesis $H_{k}$, denoted by $\pi_{k}$, is determined as

$$
\begin{aligned}
& \pi_{0}=P\left(H_{0}\right)=P\left(H_{0}, E^{\text {off }}\right)=P\left(E^{\text {off }} \mid H_{0}\right) P\left(H_{0}\right) \\
& \pi_{1}=P\left(H_{1}\right)=P\left(H_{1}, E^{\text {off }}\right)=P\left(E^{\text {off }} \mid H_{1}\right) P\left(H_{1}\right) \\
& \pi_{2}=P\left(H_{2}\right)=P\left(H_{0}, E^{\text {on }}\right)=P\left(E^{\text {on }} \mid H_{0}\right) P\left(H_{0}\right)
\end{aligned}
$$

Let the parameter $\alpha$ (called attack strength through the study) be the conditional probability regarding the presence of the fake PUEA signals in the hypothesis $H_{0}$, (i.e. $\left.\alpha=P\left(E^{o n} \mid H_{0}\right)\right)$. Thus, the above equation can be simplified to

$$
\begin{aligned}
& \pi_{0}=(1-\alpha) P\left(H_{0}\right) \\
& \pi_{1}=P\left(H_{1}\right) \\
& \pi_{2}=\alpha P\left(H_{0}\right)
\end{aligned}
$$

By considering the 3-level hypotheses, the received signal at the $i$ th sample of the $j$ th CR user, $x_{j}^{i}$, can be formulated as

$$
x_{j}^{i}= \begin{cases}n_{j}^{i} & H_{0} \\ \sqrt{\gamma_{j}} p_{j}^{i}+n_{j}^{i} & H_{1} \\ \sqrt{\lambda_{j}} e_{j}^{i}+n_{j}^{i} & H_{2}\end{cases}
$$

where $n_{j}^{i}$ is the Additive White Gaussian Noise (AWGN) at the $j$ th $\mathrm{CR}$ user. The parameters $\sqrt{\gamma_{j}} p_{j}^{i}$ and $\sqrt{\lambda_{j}} e_{j}^{i}$ are the received PU and PUEA signal with the powers $\gamma_{j}$ and $\lambda_{j}$, respectively. We assume that the noise at each sample ( $\left.n_{j}^{i}\right)$, the PU signal $\left(p_{j}^{i}\right)$, and PUEA signal sample $\left(e_{j}^{i}\right)$ are independently and identically distributed Gaussian random variables with zero mean and unit variance. We further assume that the CR users experience independent Rayleigh fading channels with the same average SNRs. This condition is relevant for CR network which is geographically far from the PU and PUEA transmitters. Thus, $\gamma_{j}$ and $\lambda_{j}$ vary from (observation) period to period while their Probability Density Functions (PDFs) are identically as exponential distribution with the average values $\bar{\gamma}$ and $\bar{\lambda}$, respectively. The parameter $\rho$ is also defined as $\rho=\bar{\lambda} / \bar{\gamma}$. Obviously, a larger value of $\rho(\rho>>1)$ indicates a more powerful PUEA. As mentioned in equation (3) and with regard to the above assumptions, the received signal, $x_{j}^{i}$, is a Gaussian distributed as [13],

$x_{j}^{i} \sim\left\{\begin{array}{lc}\mathcal{N}(0,1) & H_{0} \\ \mathcal{N}\left(0, \gamma_{j}+1\right) & H_{1} \\ \mathcal{N}\left(0, \lambda_{j}+1\right) & H_{2}\end{array}\right.$

Moreover, $M$ samples are used for local energy detection at each $\mathrm{CR}$ user during one detection interval. The observed energy of the $j$ th user, $E_{j}$, is given by

$$
E_{j}=\sum_{i=1}^{M}\left|x_{j}^{i}\right|^{2} \sim \begin{cases}a_{j} & H_{0} \\ \left(\gamma_{j}+1\right) b_{j} & H_{1} \\ \left(\lambda_{j}+1\right) c_{j} & H_{2}\end{cases}
$$

where the random variables $a_{j}, b_{j}$ and $c_{j}$ follow a central Chi-square distribution with $M$ degree of freedom. But, according to central limit theorem, if a large number of samples are considered (i.e. $M>10$ ), these random variables can be assumed to be Gaussian distributed.

In conventional Equal Gain Combining (EGC) scheme [13], in the absence of the PUEA, all of the sensing reports are summed up and compared with a predefined threshold to determine the channel status. The output signal at the FC is

$Y=\sum_{j=1}^{N} E_{j} \stackrel{\substack{H_{1} \\ H_{0}}}{<} \eta$

where $\eta$ is the global threshold and determined by the target false alarm or miss detection probability. In the presence of the PUEA, the decision statistics $Y$ is a Gaussian distributed as

$Y \sim \begin{cases}\mathcal{N}\left(\mu_{0}, \sigma_{0}\right) & H_{0} \\ \mathcal{N}\left(\mu_{1}, \sigma_{1}\right) & H_{1} \\ \mathcal{N}\left(\mu_{2}, \sigma_{2}\right) & H_{2}\end{cases}$

where

$$
\begin{array}{ll}
\mu_{0}=M N, & \sigma_{0}=2 M N \\
\mu_{1}=M N(\bar{\gamma}+1), & \sigma_{1}=2 M N(\bar{\gamma}+1)^{2} \\
\mu_{2}=M N(\bar{\lambda}+1), & \sigma_{2}=2 M N(\bar{\lambda}+1)^{2}
\end{array}
$$

Let $Q_{f a}$ and $Q_{m}$ be the probabilities of global false alarm and miss detection, respectively. Then we have

$Q_{f a}=P\left(D^{o n} \mid H_{0}\right) \quad, \quad Q_{m}=P\left(D^{o f f} \mid H_{1}\right)$

where $D^{o n}$ and $D^{\text {off }}$ mean that the FC's decisions are the presence and absence of the PU signal, respectively. To 
evaluate the performance of CSS in the presence of the PUEA and compare it to conventional method, in which the PUEA is not considered, we use global error probability $Q_{e}$. The parameter $Q_{e}$ defines probability of making a wrong decision in PU detection and it can be written as

$$
\begin{aligned}
Q_{e} & =P\left(H_{0}, D^{o n}\right)+P\left(H_{1}, D^{\text {off }}\right) \\
& =P\left(H_{0}\right) Q_{f a}+P\left(H_{1}\right) Q_{m}
\end{aligned}
$$

\section{THE PROPOSED MAXIMUM UTILITY DETECTION APPROACH}

In this section, the proposed MUD criterion is applied to find the hold hypothesis. For a 3-level test, each utility function is defined as

$$
\begin{aligned}
& U_{0}(Y)=P\left(Y \mid H_{0}\right) \pi_{0} \\
& U_{1}(Y)=P\left(Y \mid H_{1}\right) \pi_{1} \\
& U_{2}(Y)=P\left(Y \mid H_{2}\right) \pi_{2}
\end{aligned}
$$

where

$$
P\left(Y \mid H_{k}\right)=\frac{1}{\sigma_{k} \sqrt{2 \pi}} \exp \left(-\frac{\left(Y-\mu_{k}\right)^{2}}{2 \sigma_{k}^{2}}\right) \quad k=0,1,2
$$

The values of $U_{k}(Y), \quad(k=0,1,2)$ are calculated in FC and the hold hypothesis $H_{r}$ is chosen if

$$
U_{r}(Y)>U_{k}(Y) \quad \text { for all } \quad r \neq k
$$

The proposed MUD approach is summarized in Algorithm 1.
Algorithm 1: The CSS process in the presence of malicious PUEA using MUD 3-Level Hypotheses Test method
Input: Spectrum sensing reports $E_{j}$ for $j=1,2, \ldots \ldots, N$
1) Calculate $Y$ using equation (6)
2) Calculate $U_{0}(Y), U_{1}(Y)$ and $U_{2}(Y)$ using equation (10)
3) Compare $U_{0}(Y), U_{1}(Y)$ and $U_{2}(Y)$
4) $r=\arg \max _{0 \leq k \leq 2} U_{k}(Y)$

Output: Selecting $H_{r}$ as a hold hypothesis

\section{PRACTICAL CONSIDERATION}

In previous sections, we investigated collaborative sensing in the presence of a PUEA theoretically, without considering practical limitations. For instance, to find the hold hypothesis by (10), the FC needs to get the $\alpha$ value according to (2). There might be several different methods for FC to get the parameter $\alpha$ but here, we propose a method based on the mean value of received sensing reports. Two parameters $m$ and mathematical expectation of $m$ are defined as

$m=\frac{1}{N} \sum_{j=1}^{N} E_{j} \quad, \quad \mathrm{E}(m)=\frac{1}{N} \sum_{j=1}^{N} \mathrm{E}\left(E_{j}\right)$
By considering three different hypotheses $H_{0}, H_{1}$, and $H_{2}$ we have

$$
\begin{aligned}
\mathrm{E}\left(E_{j}\right) & =\mathrm{E}\left(E_{j} \mid H_{0}\right) \pi_{0}+\mathrm{E}\left(E_{j} \mid H_{1}\right) \pi_{1}+\mathrm{E}\left(E_{j} \mid H_{2}\right) \pi_{2} \\
& =M \pi_{0}+M\left(\gamma_{j}+1\right) \pi_{1}+M\left(\lambda_{j}+1\right) \pi_{2}
\end{aligned}
$$

By substituting equation (13) into equation (12), we have

$$
\mathrm{E}(m)=M \pi_{0}+M(\bar{\gamma}+1) \pi_{1}+M(\bar{\lambda}+1) \pi_{2}
$$

Finally, the value of attack strength $\alpha$ is estimated as

$$
\hat{\alpha}=\left(\mathrm{E}(m)-\psi_{1}\right) / \psi_{2}
$$

where two parameters $\psi_{1}$ and $\psi_{2}$ are defined as

$$
\psi_{1}=M P\left(H_{0}\right)+M(1+\bar{\gamma}) P\left(H_{1}\right) \quad, \quad \psi_{2}=M \bar{\lambda} P\left(H_{0}\right)
$$

\section{SIMULATION RESULTS AND DISCUSSIONS}

In the proposed system model there are $12 \mathrm{CR}$ users that use energy detection by $M=30$ samples. The channels are assumed to be Rayleigh fading. Moreover, prior probabilities $P\left(H_{0}\right)$ and $P\left(H_{1}\right)$ are assumed to be 0.8 and 0.2 , respectively. Throughout the simulations, we have depicted that there is not any PUEA signals labeled by "EGC (No Attack)" curves and the case that there is PUEA signals and the FC is not aware of the fake signals labeled by "Conventional" curves.

Figure (2) shows the convergences of attack strength for $\alpha=0.3$ and 0.7. The estimated value for $\alpha$ is converged to constant values after applying almost 300 rounds of sensing. In the simulation, the initial stage can be set as the first 500 sensing intervals where the attack strength is estimated.

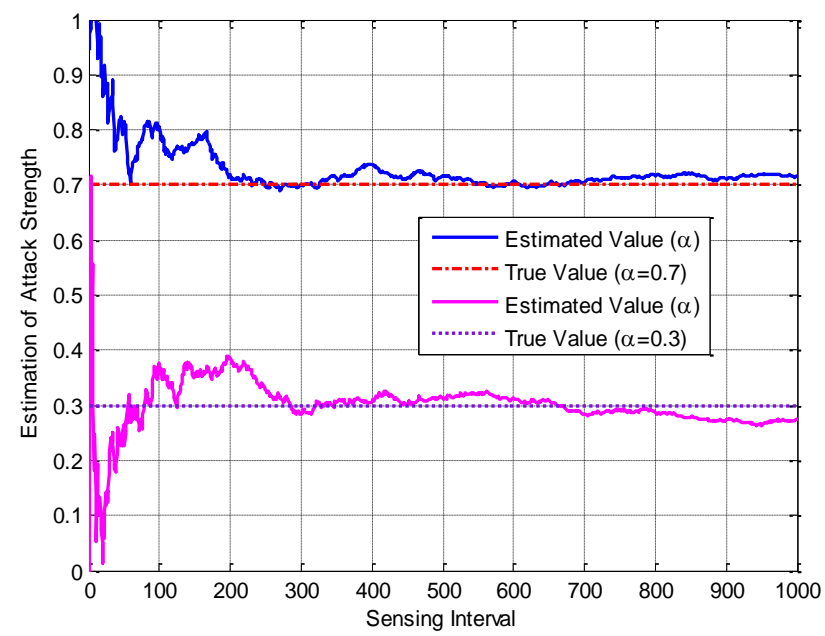

Fig. 2. The convergences of attack strength $(\alpha=0.3,0.7)$

Figure (3) shows the probability of error versus average SNR for attack strength 0.3 and 0.9 . As shown, using the proposed MUD method improves performance of CSS under PUEA signals. In conventional method, the presence of PUEA 
signals leads to high energy level in the FC. Consequently, the error probability increases with increasing the average SNR.

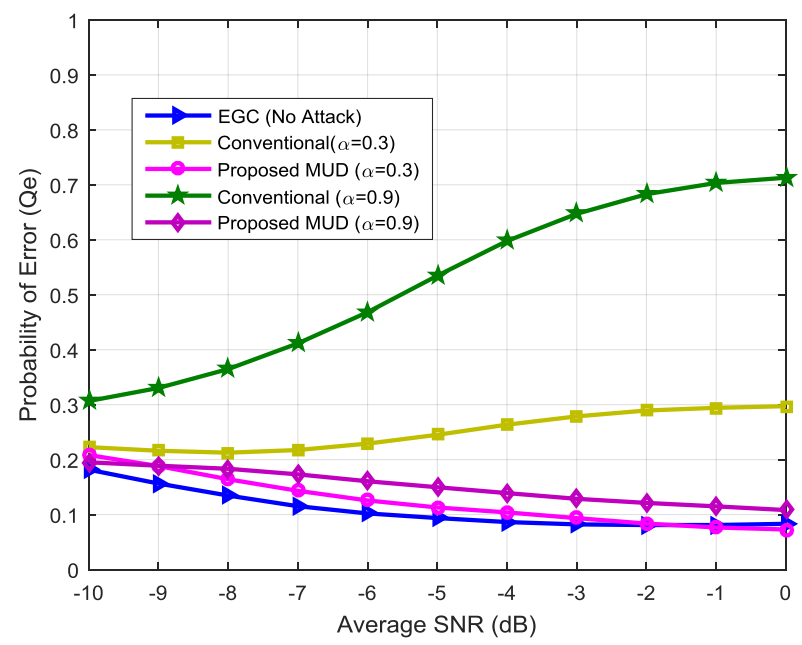

Fig. 3. Probability of error versus average SNR $(\bar{\gamma})$ with

$$
\rho=0.5
$$

Figure (4) depicts the error probability versus attack strength $\alpha$ for $\rho=1 / 2$ and $\rho=2$ in $\bar{\gamma}=-5 \mathrm{~dB}$. As shown in the figure, in conventional method, increasing $\alpha$ and $\rho$ leads to more probability of error at the FC, in contrary, by the proposed method, increasing $\alpha$ and $\rho$ causes a little changes in the rate of error probability.

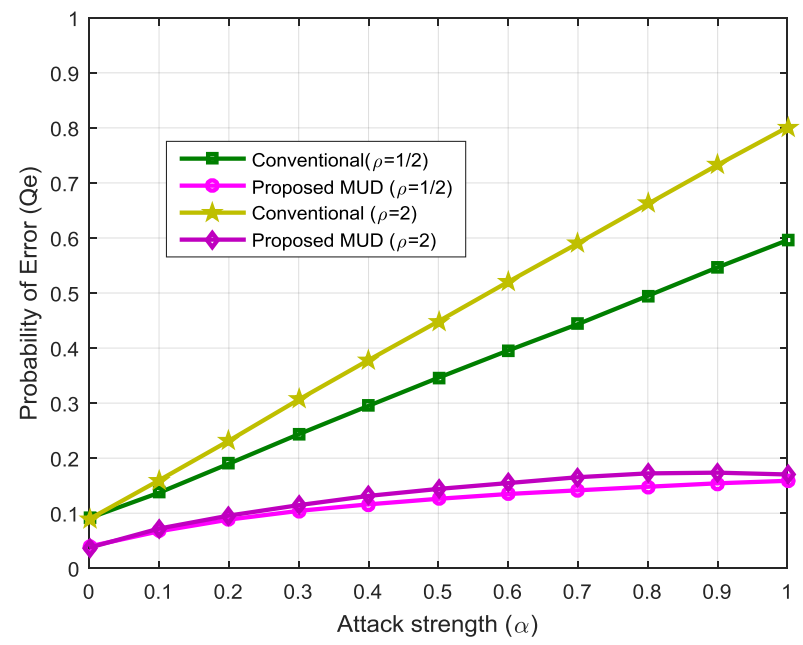

Fig. 4. Probability of error versus attack strength $(\alpha)$

Figure (5) shows the error probability versus attack parameter $\rho$ for attack strength 0.3 and 0.9 in $\bar{\gamma}=-5 \mathrm{~dB}$. As shown, using the proposed MUD method improves performance of collaborative sensing in the presence of a malicious PUEA.

\section{CONCLUSION}

In this study, a novel Collaborative Spectrum Sensing (CSS) scheme in the presence of PUEA based on Maximum Utility Detection (MUD) approach for 3-level hypothesis test was introduced. The proposed MUD method tried to find the hold hypothesis by comparing utility function of each hypothesis. The obtained results verified the effectiveness of the proposed scheme compared with conventional method.

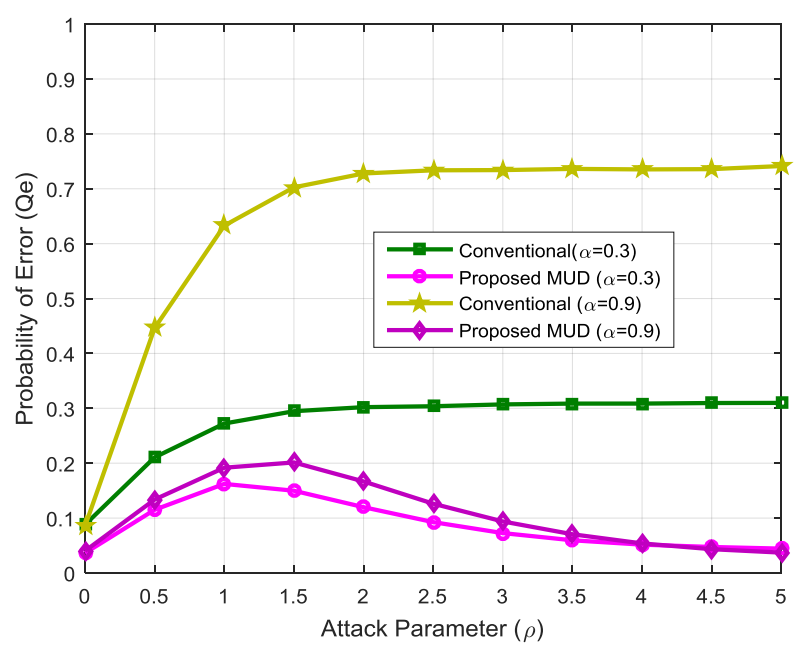

Fig. 5. Probability of error versus $\rho$

\section{REFERENCES}

[1] J. Mitola, G. Q. Maguire, "Cognitive radio: making software radios more personal," IEEE Personal Communication, vol. 6, no. 4, pp. 13-18, 1999.

[2] S. Haykin, "Cognitive radio: brain-empowered wireless communications," IEEE Journal on Selected Areas in Communications, vol. 23, no. 2, pp. 201-220, 2005.

[3] I. F. Akyildiz, W. Y. Lee, M. C. Vuran, S. Mohanty, "NeXt generation/dynamic spectrum access cognitive radio wireless networks: A survey," Computer Networks, vol. 50, no. 13, pp. 2127-2159, 2006.

[4] R. Chen, J. M. Park, "Ensuring trustworthy spectrum sensing in cognitive radio networks," In $1^{\text {st }}$ IEEE Workshop on Networking Technologies for Software Defined Radio Networks, pp. 110-119, 2006.

[5] S. Anand, Z. Jin, K. Subbalakshmi, "An analytical model for primary user emulation attacks in cognitive radio networks," In Proceeding IEEE International Dynamic Spectrum Access Networks, pp. 1-6, 2008.

[6] R. Chen, J. M. Park, J. H. Reed, "Defense against primary user emulation attacks in cognitive radio networks," IEEE Journal of Selected Area in Communications, vol. 26, no. 1, pp. 25-37, 2008.

[7] C. Chen, H. Cheng, Y. D. Yao, "Cooperative spectrum sensing in cognitive radio networks in the presence of the primary user emulation attack," IEEE Transactions on Wireless Communications, vol. 10, no. 7, pp. 2135-2141, 2011.

[8] M. Haghighat, S. M. S. Sadough, "Cooperative spectrum sensing for cognitive radio networks in the presence of smart malicious users," International Journal of Electronics and Communications (AUE), vol. 68, no. 6, pp. 520-527, 2014.

[9] Haghighat M, Sadough SMS. Smart primary user emulation in cognitive radio networks: defense strategies against radio-aware attacks and robust spectrum sensing. Transactions on Emerging Telecommunications Technologies, vol. 26, no. 9, pp. 1154-1164, 2015.

[10] R. Yu, Y. Zhang, Y. Liu, S. Gjessing and M. Guizani, "Securing Cognitive Radio Networks against Primary 
User Emulation Attacks," IEEE Network, vol. 30, no. 6, pp. 62-69, 2016.

[11] A. A. Sharifi, M. Sharifi, M. J. Musevi Niya. "Collaborative Spectrum Sensing under Primary User Emulation Attack in Cognitive Radio Networks," IETE Journal of Research, vol. 62, no. 2, pp. 205-211, 2016.

[12] A. A. Sharifi, M. Sharifi, M. J. Musevi Niya. Secure cooperative spectrum sensing under primary user emulation attack in cognitive radio networks: Attack- aware threshold selection approach. AEU - International Journal of Electronics and Communications, vol. 70, no. 1, pp. 95-104, 2016.

[13] J. Ma, G. Zhao, Y. Li, "Soft combination and detection for cooperative spectrum sensing in cognitive radio networks," IEEE Transactions on Wireless Communications, vol. 7, no. 11, pp. 4502-4507, 2008.

[14] P. K. Varshney, "Distributed Detection and Data Fusion," Springer-Verlag, 1997. 